\title{
MicroReview
}

\section{The role of the NAC protein in the nitrogen regulation of Klebsiella aerogenes}

\author{
R. A. Bender \\ Department of Biology, University of Michigan, Ann \\ Arbor, Michigan 48109-1048, USA.
}

\section{Summary}

The NAC (nitrogen assimilation control) protein from Klebsiella aerogenes is a LysR-like regulator for transcription of several operons involved in nitrogen metabolism, and couples the transcription of these $\sigma 70$-dependent operons to regulation by the $\sigma 54$ dependent NTR system. NAC activates expression of operons (e.g. histidine utilization, hut), allowing use of poor nitrogen sources, and represses expression of operons (e.g. glutamate dehydrogenase, gdh) allowing assimilation of the preferred nitrogen source, ammonium. NAC is both necessary and sufficient to activate transcription, but the expression of the nac gene is totally dependent on the central nitrogen regulatory system (NTR) and RNA polymerase carrying the $\sigma 54$ sigma factor (RNAP $\sigma 54$ ). Nitrogen starvation signals the NTR system to transcribe nac, and NAC activates the transcription of hut, put (proline utilization), and urease. NAC does not affect the transcription of RNAP $\sigma 54$-dependent operons like gInA or nifLA, which respond directly to the NTR system, but activates transcription of RNAP $\sigma 70$ dependent operons. Thus NAC acts as a bridge between RNAP $\sigma 70$-dependent operons like hut and the RNAP $\sigma 54$-dependent NTR system. The activation of operons like hut by NAC in response to nitrogen starvation is at least superficially similar to their activation by CAP-CAMP in response to carbon and energy starvation.

\section{Nitrogen regulation}

Thirty-five years ago, Neidhardt and Magasanik (1957) found that expression of the histidine-utilization (hut) operons from Klebsiella aerogenes was increased under conditions of nitrogen starvation. This increased expression was specific for pathways that yielded ammonia

Received 25 July, 1991; revised 7 August, 1991. Tel. (313) 936 2530; Fax (313) 7470884 and/or glutamate; pathways that yielded only carbon and/or energy were actually more 'catabolite repressed' under conditions of nitrogen starvation than under nitrogen excess. In $K$. aerogenes, this nitrogen regulation $(\mathrm{N}$ Reg) affects a wide array of operons involved in the fixation of ammonia into glutamate, the catabolism of amino acids, the reduction of inorganic nitrogen compounds, and the catabolism of organic compounds (Macaluso et al., 1990). A partial list of the N-Reg-responsive pathways for $K$. aerogenes is given in Table 1.

$\mathrm{N}$-Reg was not studied further at that time because of the absence of a genetic system for $K$. aerogenes. The other enteric species for which the genetics were well established were inappropriate: Escherichia coli lacks the hut operons entirely and Salmonella typhimurium does not show N-Reg of hut. The discovery of a transducing phage for $K$. aerogenes led to a renewal of $\mathrm{N}$-Reg studies (Prival and Magasanik, 1971) and to the discovery of the central role of the nitrogen regulatory system (NTR) system in N-Reg. Many groups around the world have contributed to our current understanding of the NTR system in the enteric bacteria, but three groups in particular have served as continuing foci for this study: those of Magasanik (working mainly with E. coli), Kustu (working mainly with $S$. typhimurium), and the Nitrogen Fixation Unit at Sussex, UK (working mainly with K. pneumoniae).

\section{The GLN system}

The preferred nitrogen source for $K$. aerogenes is ammonium. All other nitrogen compounds result in slower growth (when glucose is the carbon source) and thus are growth-rate-limiting. However, $K$. aerogenes cannot respond directly to ammonium itself. Ammonium has no effect on N-Reg unless it can be converted to glutamine (Bender and Magasanik, 1977). Thus, a discussion of $\mathrm{N}$ Reg must begin with the regulation of glutamine metabolism described by Stadtman and his colleagues (Ginsburg and Stadtman, 1973). The GLN system, the regulatory network that controls the rate of assimilation of ammonium into the amide position of glutamine, is built around a small regulatory protein, $\mathrm{P}_{\|}$(encoded by $g / n B$ ), which can exist in two forms - unmodified $\left(P_{11}\right)$ or modified by the covalent attachment of one UMP residue to each subunit $\left(\mathrm{P}_{11}-\mathrm{UMP}\right)$. The uridylyltransferase 


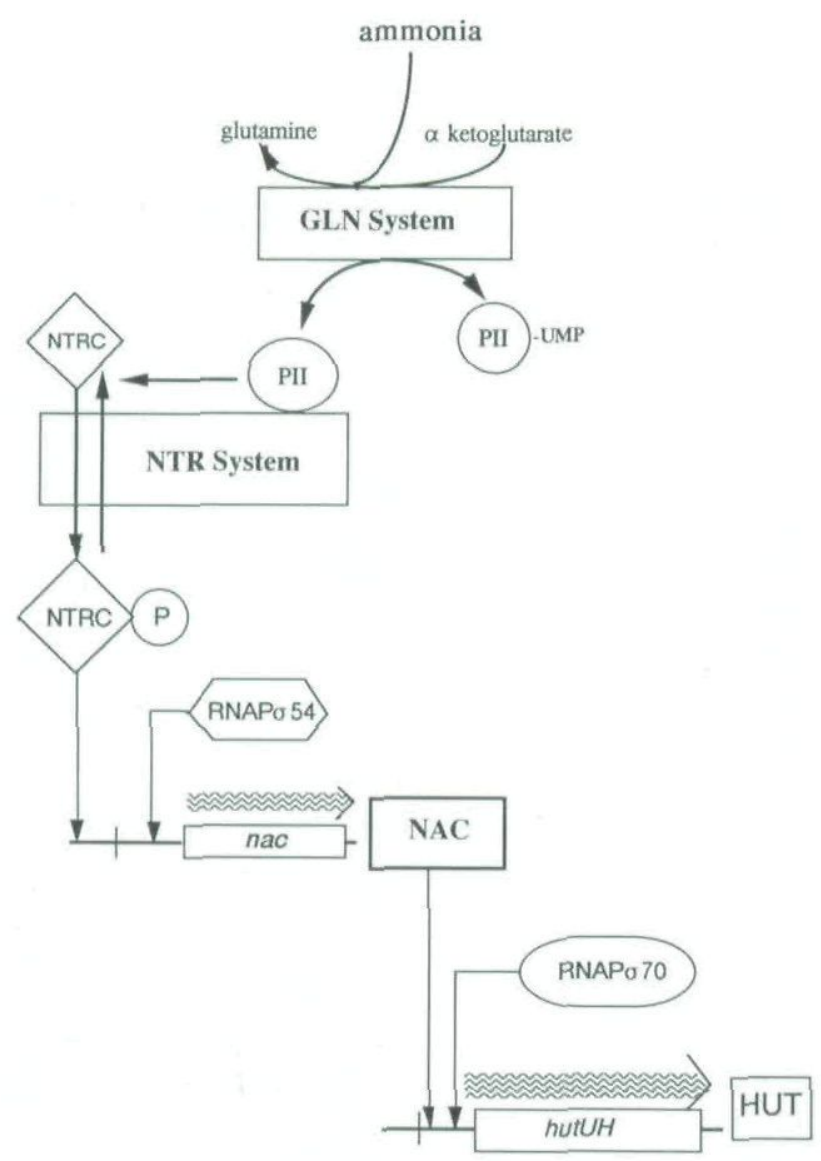

Fig. 1. The role of the NAC protein in nitrogen regulation of HUT (histidine utilization) enzyme formation. Three levels of control can be distinguished. (i) Addition of ammonia leads to accumulation of glutamine, which in turn causes $P_{\mid 1}$ to accumulate in its unmodified form. (ii) Unmodified $P_{11}$ greatly stimulates dephosphorylation of NTRCphosphate (NTRC-P) to its inactive form (NTRC), thus eliminating expression of the nac gene by RNA Polymerase carrying the $\sigma 54$ subunit (RNAP 554 ). (iii) In the absence of NAC, hut transcription by RNAP $\sigma 70$ is not activated. Thus addition of ammonia leads to loss of hut expression. Conversely, in the absence of ammonia, the glutamine pool declines and $P_{11}$ is modified to $P_{11}$-UMP. $P_{11}$-UMP does not stimulate dephosphorylation of NTRC-P so active NTRC-P accumulates and stimulates transcription of nac by RNAP $\sigma 54$. NAC protein accumulates and stimulates transcription of the hut operons by RNAP $\sigma 70$. Thus when ammonia (glutamine) is removed, $P_{11}$ is inactivated to $P_{11}$-UMP, NTRC is activated to NTRC-P, NAC is produced, and hut operon expression is activated.

(encoded by $g \ln D$ ) responsible for modifying $\mathrm{P}_{\|}$ (reversibly) is regulated by the intracellular concentrations of glutamine (indicating $\mathrm{N}$ excess) and 2-ketoglutarate (indicating $\mathrm{N}$ starvation). When nitrogen is limiting, UMP residues are added and $P_{\|}-U M P$ accumulates. When nitrogen is abundant, UMP residues are removed and $\mathrm{P}_{\|}$accumulates. When nitrogen is in excess, $\mathrm{P}_{\|}$signals an inactivation (by adenylylation) of glutamine synthetase, thus reducing the rate of glutamine formation. When nitrogen is in short supply, $\mathrm{P}_{11}$-UMP signals the reactivation (by deadenylylation) of glutamine synthetase, thus increasing the rate of glutamine formation. Thus $P_{\|}$ plays a homeostatic role in the GLN system, keeping the rate of glutamine synthesis balanced against the supply of ammonia and the biosynthetic demand for glutamine. But $P_{\|}$also plays a role in regulating transcription by interaction with the NTR system.

\section{The NTR system}

The NTR of enteric bacteria is the subject of numerous recent reviews (e.g. Kustu et al., 1986; Magasanik and Neidhardt, 1987; Merrick, 1988), so only a simplified outline is presented here. RNA polymerase carrrying the 54 $\mathrm{kDa}$ sigma subunit (RNAP $\sigma 54$ ) differs from the more common RNAP $\sigma 70$ in two ways: RNAP $\sigma 54$ recognizes a very different promoter sequence from RNAP $\sigma 70$, and RNAP $\sigma 54$ seems unable to form open complexes at its promoters unless it interacts with a specific activator, i.e. an enhancer-binding protein. The NTR system itself consists of such an activator protein (called variously NTRC or $\mathrm{NR}_{4}$ ) and a specific phosphotransferase (called variously NTRB or $\mathrm{NR}_{11}$ ) capable of phosphorylating and dephosphorylating the activator. Although both the phosphorylated and non-phosphorylated forms of the activator (NTRC-P and NTRC in Fig. 1) can recognize enhancer sequences on the DNA and bind there, only NTRC-P can interact with RNAP $\sigma 54$ to cause open complex formation. In the absence of other proteins, the phosphotransferase

Table 1. Pathways known to be responsive to $\mathrm{N}$-Reg in $\mathrm{K}$. aerogenes.

$\mathrm{Nac} ?^{\mathrm{a}}$

Ammonia assimilation

Glutamine synthetase ( $g \ln A)$

Glutamate synthase

Glutamate dehydrogenase ( $g d h)$

( $\mathrm{NH}_{4} \rightarrow$ glutamine) (glutamine $\rightarrow$ glutarnate)

Amino acid catabolism

Histidine utilization ( $($ nut $)$

Proline utilization (put)

Tryptophan utilization

Asparagine utilization

Ornithine utilization

$\left(\mathrm{NH}_{4} \rightarrow\right.$ glutamate)

$-$

$+$

Inorganic nitrogen compounds

Dinitrogen fixation (nifL)

Nitrate reduction (assimilatory)

(histidine $\rightarrow \mathrm{NH}_{4}+$ glutamate) + (proline $\rightarrow$ glutamate) (tryptophan $\rightarrow \mathrm{NH}_{4}$ ) (asparagine $\left.\rightarrow \mathrm{NH}_{4}\right)^{\mathrm{b}}$ (ornithine $\rightarrow$ glutarnate)

$+$

Organic nitrogen compounds

Urea utilization (ure)

$\left(\mathrm{N}_{2} \rightarrow \mathrm{NH}_{4}\right)$

$\left(\mathrm{NO}_{3} \rightarrow \mathrm{NH}_{4}\right)$

$\left(\right.$ urea $\rightarrow \mathrm{NH}_{4}$ )

$+$

a. A plus in this column indicates that NAC is required for N-Reg; every system known to be responsive to N-Reg requires the NTR system for NReg.

b. It is not known how $K$. aerogenes derives $\mathrm{NH}_{4}$ from the aspartate that is also formed from this reaction; nor is it known how glutamate is catabolized to $\mathrm{NH}_{4}$. 
(NTRB) can catalyse the phosphorylation of NTRC as well as allow the slow autodephosphorylation of NTRC-P: thus NTRC-P (the active form) accumulates. The connection between the NTR proteins and N-Reg comes through $P_{\| 1}$. Unmodified $P_{\| \prime}$ (signalling $N$ excess) greatly stimulates the dephosphorylating activity of NTRB; $\mathrm{P}_{||}$-UMP (signalling $\mathrm{N}$ starvation) may be inert with respect to NTRB. Thus when $P_{\| \prime}$ is unmodified, inactive NTRC accumulates; when $P_{11}$ is modified to $P_{11}-U M P$, active NTRC-P accumulates and RNAP $\sigma 54$ transcribes actively.

This model of NTR control has been tested using purified components in vitro at both ginA (glutamine synthetase) promoter and nif (dinitrogen fixation) and found to be sufficient to explain N-Reg of these promoters. It was tempting to extend the model to explain $\mathrm{N}$-Reg of all the systems in Table 1, particularly in view of the fact that mutants lacking either the activator (NTRC-P) or the $\sigma 54$ subunit of RNAP $\sigma 54$ also lacked N-Reg of every system is Table 1 (Macaluso et al., 1990). However, although the NTR system in necessary for N-Reg of all the pathways in Table 1, it is sufficient only for some. The remaining pathways, e.g. hut , also require the NAC (nitrogen assimilation control) protein for their N-Reg.

\section{The NAC system}

Mutants that lack NAC because of insertions in the nac gene of $K$. aerogenes have lost $\mathrm{N}$-Reg of some, but not all, pathways (Bender et al., 1983; Macaluso et al., 1990). In particular, the activation of hut and the proline utilization (put) system is lost in NAC-defective strains, but the activation of $g \ln A$ and nifL operons remains intact in NACdefective strains. Consistent with this difference, both the hut and put operons are transcribed by RNAP $\sigma 70$ (Nieuwkoop et al., 1988; Chen and Maloy, 1991) whereas the gInA and nifL operons are transcribed by RNAP $\sigma 54$. Thus NAC is necessary for N-Reg of hut, put, and perhaps other operons transcribed by RNAP $\sigma 70$.

NAC is also sufficient for activation of hut and put expression. When an isopropyl- $\beta$-D-thiogalactoside (IPTG)-inducible tac promoter (Chow and Berg, 1988) was inserted between the nac promoter and the nac coding sequences, NAC-dependent operons like hut and put were activated by IPTG, not by $\mathrm{N}$ starvation (A. Schwacha and R. A. Bender, unpublished). This IPTG induction of hut and put occurred even in the presence of excess ammonia, showing that if NAC was formed, hut and put were activated no matter what the metabolic state of the cell with respect to nitrogen. In fact, when NAC was produced from this IPTG-inducible promoter, even cells lacking the NTR system (because of mutations eliminating NTRC or $\sigma 54$ ) showed activation of hut and put. Thus NAC appears sufficient for activation and the only role of the NTR system is to control expression of the nac gene.

\section{NAC formation is controlled by the NTR system}

Fusion of the nac promoter with the lacZgene allowed us to monitor $\beta$-galactosidase as a measure of nac gene expression (Macaluso et al., 1990). Strains with a wildtype NTR system showed strong N-Reg of nac expression. Strains lacking NTRC or $\sigma 54$ were unable to derepress nac, even under severe $\mathrm{N}$ starvation. Strains constitutive for the NTR system were constitutive for nac as well. The sequence of the DNA region upstream of the nac coding sequence shows a strong match to the consensus RNAPr54-dependent promoters (A. Schwacha and R. A. Bender, unpublished). About 120 bp further upstream is a match to a consensus NTRC-binding site.

NTR control of nac expression has been directly demonstrated by in vitro transcription of nac with purified components (A. Ninfa and R. A. Bender, unpublished). A cloned nac promoter was very efficiently transcribed by RNAP $\sigma 54$ if NTRC-P was present. Omission of NTRC-P or substitution of NTRC for NTRC-P eliminated transcription of nac. As expected, the amount of NTRC-P required for nac transcription was somewhat higher than that required for $g \ln A$ transcription. Thus we assume that nac is an operon like nifLA whose transcription is directly regulated by the NTR system and whose function is the regulation of a subfamily of nitrogen-responsive operons, either directly or (as is the case with NIFL) in response to other regulatory signals.

\section{What do we know about NAC?}

Studies of the nac gene (A. Schwacha and R. A. Bender, unpublished) and the NAC protein (T. J. Goss and R. A. Bender, unpublished) have given us much information about NAC. The nac gene was cloned and sequenced. The deduced amino acid sequence of NAC immediately identified NAC as a member of a family of regulatory proteins known as the LysR family (Henikoff et al., 1988), supporting the notion that NAC is a regulatory protein.

The NAC protein was purified to near homogeneity ( $T$. J. Goss and R. A. Bender unpublished) and is probably a dimer in solution. Purified NAC bound to hutUp DNA in gel mobility shift assays. Purified NAC also stimulated RNAP $\sigma 0$-mediated transcription from hutUp, the promoter of the hutUH operon, just as CAP-CAMP does. In contrast to other LysR family regulators, NAC may have only one conformation. NAC produced in vivo from a tac promoter activated hut, put and urease gene transcription whether the cells were starved for nitrogen or not. Moreover, NAC made under $\mathrm{N}$ starvation and $\mathrm{N}$ excess were equally able to bind hutUp DNA, and the presence of such traditional $\mathrm{N}$-Reg signals as 2-ketoglutarate and glutamine did not seem to affect NAC's ability to activate RNAP $\sigma 70$-mediated transcription in a purified system. 
To summarize, we know that NAC is necessary and sufficient to explain the transcriptional activation of the hut operons (and several others) in response to N-Reg. Three clear questions remain unanswered: (i) do other organisms have the NAC; (ii) how does NAC activate transcription; and (iii) why does $K$. aerogenes need NAC when NTR seems able to do the job?

\section{NAC in other bacteria}

Clearly $K$, aerogenes has NAC. Equally clearly, S. typhimurium does not. S. typhimurium cannot activate hut expression in response to N-Reg (Magasanik, 1978), even though it has a normal NTR system (Kustu et al., 1986). When the hut genes from S. typhimurium were moved to $K$. aerogenes, they responded to N-Reg (Goldberg et al., 1976) as long as the $K$. aerogenes used was nac $^{+}$(Best and Bender, 1990). In a complementary experiment, the cloned nac gene from $K$. aerogenes was moved into $S$. typhimurium, and the resulting strain showed N-Reg of hut expression (Best and Bender, 1990). Thus wild-type S. typhimurium lacks an active NAC product. The situation with $E$. coli is less clear, but it appears that $E$. coli has an active NAC. Wild-type E. coli lacks the hut operons, but when the hut genes from $K$. aerogenes were moved into $E$. coli they responded to $\mathrm{N}$ Reg in E. coli, albeit less dramatically (Goldberg et al., 1976). Moreover, introduction of the cloned nac gene from $K$. aerogenes into $E$. coli did not increase hut expression (our unpublished results). Preliminary Southern blots detected an $E$. coli DNA sequence with similarity to the $K$. aerogenes nac gene but found no such sequence in $S$. typhimurium (W. R. Muse and R. A. Bender, unpublished). Thus $S$. typhimurium appears to have lost nac. It also could be argued that $E$. coli is in the process of losing the NAC system, since the operons known to be NACdependent in $K$. aerogenes are either absent from E. coli (hut, ure) or have lost their ability to respond to the N-Reg signal (totally for gdh and almost totally for put). Nonenteric bacteria (e.g. the pseudomonads and the rhizobia) have hut operons that are quite similar to hut from $K$. aerogenes both in sequence similarity and in their response to $\mathrm{N}$-Reg. To date, we have no information on the existence of a NAC analogue outside the enterics, but the overall sequence similarity between the hut operons of Pseudomonas putida and those of $K$. aerogenes leads one to speculate that NAC might also be found outside the enterics, particularly since LysR family members are common outside the enterics.

\section{How does NAC activate transcription?}

We have few data that address this question. We know that NAC binds specifically to the promoter regions of hut,

\section{ANATOMY OF THE hut OPERONS}

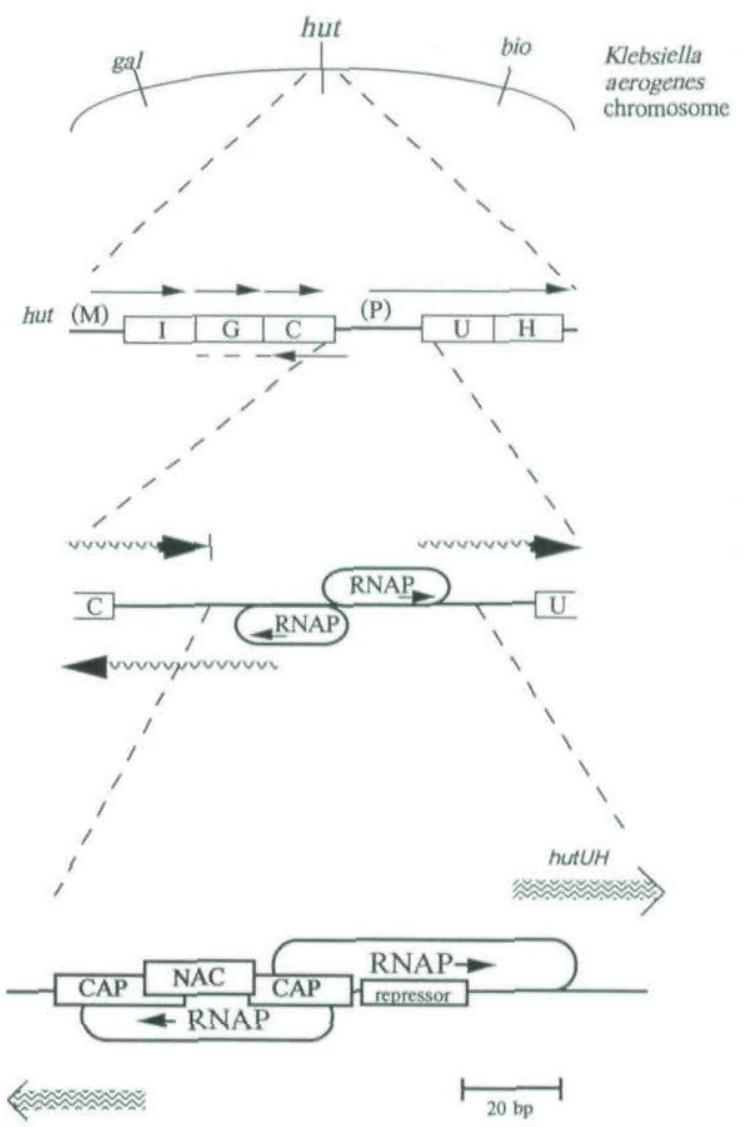

Fig. 2. The 'anatomy' of the hut operons. Line 1 indicates that the hut gene cluster lies between gal and bio. Line 2 (not to scale) shows the locations of the genes in the cluster. The transcription units (Schwacha et al., 1990) are indicated by arrows. Line 3 shows the overlap between the promoter of the hutUH operons and a promoter of unknown function reading opposite hut transcription. Line 4 shows the location of the known binding sites within the hutUH promoter region. The extent of the sites was determined by deletion analysis using a functional assay (RNA Polymerase), gel mobility shift assay (two CAP-CAMP sites and a NAC site) or an in vivo titration assay (repressor). The RNAP sites have been confirmed by an exonuclease III 'footprint' assay (Nieuwkoop and Bender, 1988)

put and ure, the three operons whose transcription is activated by NAC (T. J. Goss and R. A. Bender, unpublished). For hut, the NAC-binding site is centered at about -64 relative to the start of transcription (Fig. 2), a position that mimics the site of CAP-cAMP binding in the lac operon of E. coli (Reznikoff and Abelson, 1978). The NAC-binding site in put (T. J. Goss and R. A. Bender, unpublished) is in a similar position relative to the transcriptional start of the putP gene (Chen and Maloy, 1991); thus we assume that position may be important. The NAC-binding site in ure is about $900 \mathrm{bp}$ upstream from the ure $A$ coding region, but the transcription start of the operon has not yet been identified. 
Returning to hut, we note that the two CAP-cAMP sites centered at -42.5 and -81.5 (R. Osuna and R. A. Bender, manuscript in preparation) would cause a double bend in the promoter that would be expected to perturb at least the -35 region of the hutUH promoter. Since the NAC site is almost exactly in the middle of these two CAP sites, it is tempting to speculate that the binding of a single NAC molecule at -64 might bend the DNA into a structure resembling that formed by two CAP-CAMP complexes bound at -42 and -81 (Fig. 2). We have not yet demonstrated that CAP-CAMP can bend DNA in the hutUH promoter, much less that NAC can. Still, the model is attractive for three reasons. (i) It explains how two different positive regulators, bound at two different sites, can activate the same RNAP $\sigma 70$. (ii) Several of the known NAC. binding sites have a run of five or more $A$ residues in their central region interrupted by a single base pair. If this base pair were eliminated, one might see a 'natural bend' and it is tempting to view NAC as 'neutralizing' this base pair. (iii) The bending model is the easiest model to disprove: either NAC bends the DNA or it does not.

Besides activating operons involved in catabolism of poor nitrogen sources, NAC also represses transcription of operons involved in the assimilation of ammonium. For example, glutamate dehydrogenase $(g d h)$ is strongly repressed by NAC (Bender et al., 1983; Macaluso et al., 1990). NAC bound to a DNA fragment from a cloned gdh gene (T. J. Goss and R. A. Bender, unpublished), but neither the precise binding site nor the location of the $g d h$ promoter has yet been determined. It is easy to imagine that NAC could interfere with the binding of RNAP or some other factor essential for $g d h$ transcription.

\section{Why does $\mathrm{K}$. aerogenes need NAC?}

The paradox is simple: the NTR system regulates the expression of several operons directly in response to $\mathrm{N}$ Reg. Interposing another regulator between the NTR system and (e.g.) hut makes sense if another degree of regulation is added, but we have been unable to find any such regulation. When NAC is made, hut is made; when NAC is not made, hut is not made. Why not just regulate hut directly with NTR? Two features of the system offer a hint - one physiological and one structural. The IPTGinducible nac gene allowed us to vary the level of nac expression and showed clearly that ure, gdh, and hut have different sensitivities to NAC (A. Schwacha and R. A. Bender, unpublished). ure is almost fully derepressed before gdh repression becomes appreciable, and gdh is almost fully repressed before hut derepression becomes appreciable. Thus NAC may serve as a signal amplifier in a cascade control, conceptually similar to the cascade control described for glutamine synthetase regulation (Ginsburg and Stadtman, 1973).
The structural argument for NAC follows from the observation that hut and put are transcribed by RNAP $\sigma 70$ and are arranged in clustered operons with hutC immediately upstream of hutUH (Schwacha and Bender, 1990) and putA immediately upstream of putP (Chen and Maloy, 1991). Adding an extra (RNAPo54-specific) promoter to either the hutUH or the putP promoter region might not interrupt any essential arrangement, but the need to put an enhancer 100-200 bp upstream of the RNAP $\sigma 54$-specific promoter would require insertions into coding regions. Thus attempts to recruit RNAP $\sigma 70$ dependent operons into an RNAPø54-dependent regulatory system might prove too complex. Adding a NAC site might enlarge the promoter somewhat, but would not require alterations in nearby coding regions. Thus, in evolutionary terms, we view NAC as an agent for recruiting RNAP $\sigma 70$-dependent operons into an RNAP $\sigma 54-d e p e n-$ dent regulatory system.

All the NAC-dependent operons known to date have secondary roles. hut and put are used to provide carbon and energy and are regulated by CAP-CAMP in all three enterics. Glutamate dehydrogenase is important both for amino acid biosynthesis and for osmoregulation; it is regulated in response to both these signals in all three enterics. Even urease has a secondary role (agmatine degradation in $K$. aerogenes) but its regulation (in $K$. aerogenes) is strictly by N-Reg. Even where the NAC regulation is absent (e.g. S. typhimurium) these other controls remain active. Thus it is attractive to imagine that these operons originally evolved for other functions and that the development of a NAC system allowed them to be co-opted into the nitrogen regulatory response of $K$. aerogenes. In summary, we would argue that NAC is a transcriptional activator that couples RNAP $\sigma 70$-dependent operons to the RNAP $\sigma 54-d e p e n d e n t$ NTR system.

\section{Acknowledgements}

This work was supported by a Public Helath Service (GM47156) from the National Institutes of Health. I am especially grateful to my colleagues Thomas J. Goss and Anthony Schwacha (this laboratory) and Alex Ninfa (Wayne State University) for allowing me to discuss their unpublished work.

\section{References}

Bender, R.A., and Magasanik, B. (1977) J Bacteriol 132: 100105.

Bender, R.A. et al. (1983) J Bacteriol 156: 444-446.

Best, E.A., and Bender, R.A. (1990) J Bacteriol 172: 70437048.

Chen, L.-M., and Maloy, S. (1991) J Bacteriol 173: 783-790.

Chow, W.-Y., and Berg, D.E. (1988) Proc Natl Acad Sci USA 85: $6468-6472$.

Ginsburg, A., and Stadtman, E.R. (1973) In The Enzymes of 
Glutamine Metabolism. Prusiner, S., and Stadtman, E.R. (eds). New York: Academic Press, pp. 9-43.

Goldberg, R.B. et al. (1976) J Bacteriol 127: 114-119.

Henikoff, S. et al. (1988) Proc Natl Acad Sci USA 85: 66026606.

Kustu, S. et al. (1986) In Regulation of Gene Expression. Symposium of the Society for General Microbiology. Booth, I.R., and Higgins, C.R. (eds). Cambridge: Cambridge University Press, pp.139-154.

Macaluso, A. et al. (1990) J Bacteriol 172: 7249-7255.

Magasanik, B. (1978) In The Operon. Miller, J.H., and Reznikoff, W.S. (eds). Cold Spring Harbor, New York: Cold Spring Harbor Laboratory Press, pp. 373-387.

Magasanik, B., and Neidhardt, F.C. (1987) In Escherichia coli and Salmonella typhimurium. Neidhardt, F.C. (ed.). Washington, D.C., American Society for Microbiology, pp. 1318-1325.
Merrick, M. (1988) In The Nitrogen and Sulphur Cycles. Cole, J.A., and Ferguson, S.J. (eds). Cambridge: Cambridge University Press, pp. 331-361.

Neidhardt, F.C., and Magasanik, B. (1957) J Bacteriol 73: 253-259.

Nieuwkoop, A.J., and Bender, R.A. (1988) J Bacteriol 170: 4986-4990.

Nieuwkoop, A.J. et al. (1988) J Bacteriol 170: 2240-2246.

Prival, M.J., and Magasanik, B. (1971) J Biol Chem 246: 6288-6296.

Reznikoff, W.S., and Abelson, J.N. (1978) In The Operon. Miller, J.H., and Reznikoff, W.S. (eds). Cold Spring Harbor, New York: Cold Spring Harbor Laboratory Press, pp. 221-243.

Schwacha, A., and Bender, R.A. (1990) J Bacteriol 172: $5477-$ 5481.

Schwacha, A. et al. (1990) J Bacterio/ 172: 5991-5998. 
This document is a scanned copy of a printed document. No warranty is given about the accuracy of the copy. Users should refer to the original published version of the material. 